\title{
Fermi Surface Mapping and the Nature of Charge-Density-Wave Order in the Kagome Superconductor $\mathrm{CsV}_{3} \mathrm{Sb}_{5}$
}

\author{
Brenden R. Ortiz, ${ }^{1, *, \dagger}$ Samuel M. L. Teicher $\odot,{ }^{1, \dagger}$ Linus Kautzsch $\odot,{ }^{1}$ Paul M. Sarte, ${ }^{1}$ Noah Ratcliff, ${ }^{1}$ \\ John Harter, ${ }^{1}$ Jacob P. C. Ruff, ${ }^{2}$ Ram Seshadri, ${ }^{1}$ and Stephen D. Wilson $\oplus^{1, \$}$ \\ ${ }^{1}$ Materials Department and California Nanosystems Institute, University of California Santa Barbara, \\ Santa Barbara, California 93106, USA \\ ${ }^{2}$ CHESS, Cornell University, Ithaca, New York 14853, USA
}

(Received 16 April 2021; revised 18 August 2021; accepted 16 September 2021; published 11 November 2021)

\begin{abstract}
The recently discovered family of $A \mathrm{~V}_{3} \mathrm{Sb}_{5}(A: \mathrm{K}, \mathrm{Rb} \mathrm{Cs})$ kagome metals possess a unique combination of nontrivial band topology, superconducting ground states, and signatures of electron correlations manifest via competing charge density wave order. Little is understood regarding the nature of the charge density wave (CDW) instability inherent to these compounds and the potential correlation with the onset of a large anomalous Hall response. To understand the impact of the CDW order on the electronic structure in these systems, we present quantum oscillation measurements on single crystals of $\mathrm{CsV}_{3} \mathrm{Sb}_{5}$. Our data provide direct evidence that the CDW invokes a substantial reconstruction of the Fermi surface pockets associated with the vanadium orbitals and the kagome lattice framework. In conjunction with density functional theory modeling, we are able to identify split oscillation frequencies originating from reconstructed pockets built from vanadium orbitals and Dirac-like bands. Complementary diffraction measurements are further able to demonstrate that the CDW instability has a correlated phasing of distortions between neighboring $\mathrm{V}_{3} \mathrm{Sb}_{5}$ planes, and the average structure in the CDW state is proposed. These results provide critical insights into the underlying CDW instability in $A \mathrm{~V}_{3} \mathrm{Sb}_{5}$ kagome metals and support minimal models of CDW order arising from within the vanadium-based kagome lattice.
\end{abstract}

DOI: $10.1103 /$ PhysRevX.11.041030

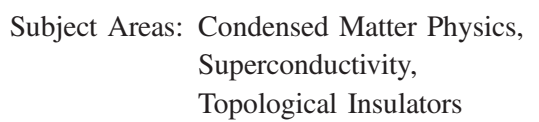

\section{INTRODUCTION}

While kagome insulators are traditionally sought as potential hosts of quantum spin liquid states and laboratories for highly frustrated magnetism [1-5], kagome metals are equally interesting due to their potential to host topologically nontrivial electronic states interwoven with local electronic symmetry breaking. At a single-orbital tight-binding level, the kagome structural motif naturally gives rise to an electronic structure with Dirac points and a flat band, which, together, provide the potential for an interplay between topologically nontrivial surface states and substantial electron correlation effects. A wide array of instabilities have been predicted, including bond density

\footnotetext{
*ortiz.brendenr@gmail.com

${ }^{\dagger}$ These authors contributed equally to this work.

¥stephendwilson@ucsb.edu
}

Published by the American Physical Society under the terms of the Creative Commons Attribution 4.0 International license. Further distribution of this work must maintain attribution to the author(s) and the published article's title, journal citation, and DOI. wave order [6,7], charge fractionalization [8,9], spin liquid states [10], charge density waves (CDW) [11], and superconductivity $[6,12]$.

The electron filling within the kagome framework controls the formation of a wide variety of predicted electronic instabilities. For band fillings near 5/4 electrons per band [6,13-16], a van Hove singularity is formed at the Fermi level due to the presence of saddle points along the zone edge. Excitations between these saddle points can lead to CDW order, and, in some limits, unconventional superconductivity. The recently discovered class of $A V_{3} \mathrm{Sb}_{5}$ ( $A$ : $\mathrm{K}, \mathrm{Rb} \mathrm{Cs})$ kagome metals [17] are potential realizations of this physical mechanism, with each member exhibiting thermodynamic anomalies associated with CDW order [18-23] followed by the onset of superconductivity at lower temperatures $[20,21,24]$. While there are multiple gaps identified $[25,26]$ with both evidence of $s$-wave pairing [27] and evidence of nodal quasiparticles [28], the interplay between superconductivity and the CDW state can, in principle, lead to unconventional behavior even in a fully gapped superconducting state [29].

The CDW instability in $A \mathrm{~V}_{3} \mathrm{Sb}_{5}$ compounds seemingly competes with superconductivity $[30,31]$ and presages the 
formation of a potentially unconventional superconducting ground state [21,31]. However, the microscopic origin of the CDW remains an open question. Concomitant to the onset of CDW order, an exceptionally large anomalous Hall effect (AHE) appears [32,33], despite the absence of detectable local moments or magnetic correlations [34]. While the normal-state electronic structure is a $\mathbb{Z}_{2}$ topological metal $[20,21]$ and topologically protected surface states are predicted close to the Fermi level [20], below the CDW transition, recent scanning tunneling microscopy (STM) data [18] and theoretical proposals [16] have suggested the formation of a chiral CDW order parameter. This chiral CDW, endemic to the kagome lattice, is proposed to break time-reversal symmetry and generate a large Berry curvature, potentially accounting for the AHE. To date, however, data directly linking the onset of CDW order with reconstruction of vanadium orbitals associated with the kagome lattice in $A \mathrm{~V}_{3} \mathrm{Sb}_{5}$ are lacking. Similarly, the applicability of minimal, single-orbital, tightbinding kagome models in multiband $A \mathrm{~V}_{3} \mathrm{Sb}_{5}$ compounds remains an open question.

Specifically, STM and diffraction experiments have observed charge order with an in-plane $\mathbf{q}=(0.5,0.5)$ wave vector in $\mathrm{KV}_{3} \mathrm{Sb}_{5}$ [18] and $\mathrm{CsV}_{3} \mathrm{Sb}_{5}$ corresponding to $3 \mathbf{Q} \mathrm{CDW}$ order. A kagome "breathing" mode can give rise to candidate distortions such as the Star of David (see Fig. 1) and its inverse structure [35], and recent studies have shown strong electron-phonon coupling in $\mathrm{KV}_{3} \mathrm{Sb}_{5}$ promoting such a distortion [36]. Native electronic instabilities promoting CDW order along this wave vector have long been predicted in kagome models at select fillings near van Hove singularities [6,13-16], suggesting that a minimal model built around the kagome planes of these materials may capture the essential physics governing their unconventional electronic properties. Notably, additional features such as unidirectional charge stripe order [19] also seemingly coexist with the $3 \mathbf{Q}$ CDW state, further connecting the underlying interactions to stripe or nematic instabilities predicted within a kagome network [16].

Here, we investigate the origins of the $3 \mathbf{Q} \mathrm{CDW}$ order in $A \mathrm{~V}_{3} \mathrm{Sb}_{5}$ kagome compounds via the study of Shubnikovde Haas $(\mathrm{SdH})$ quantum oscillations in magnetotransport data of $\mathrm{CsV}_{3} \mathrm{Sb}_{5}$, which has the most pronounced CDW instability within the family. By correlating quantum oscillation data with DFT models of the breathing distortion of the kagome lattice, we are able to observe the effect of the CDW on the electronic structure. Specifically, we demonstrate that a series of low-frequency quantum oscillations originate from CDW-reconstructed vanadium orbitals and exhibit transport consistent with the Dirac-like features (high mobility and low cyclotron mass) of the kagome lattice. The multiplicity and frequencies associated with these vanadium orbits are shown to originate from a reconstructed Fermi surface with small pockets linked to folded, vanadium-dominated bands. We further demonstrate

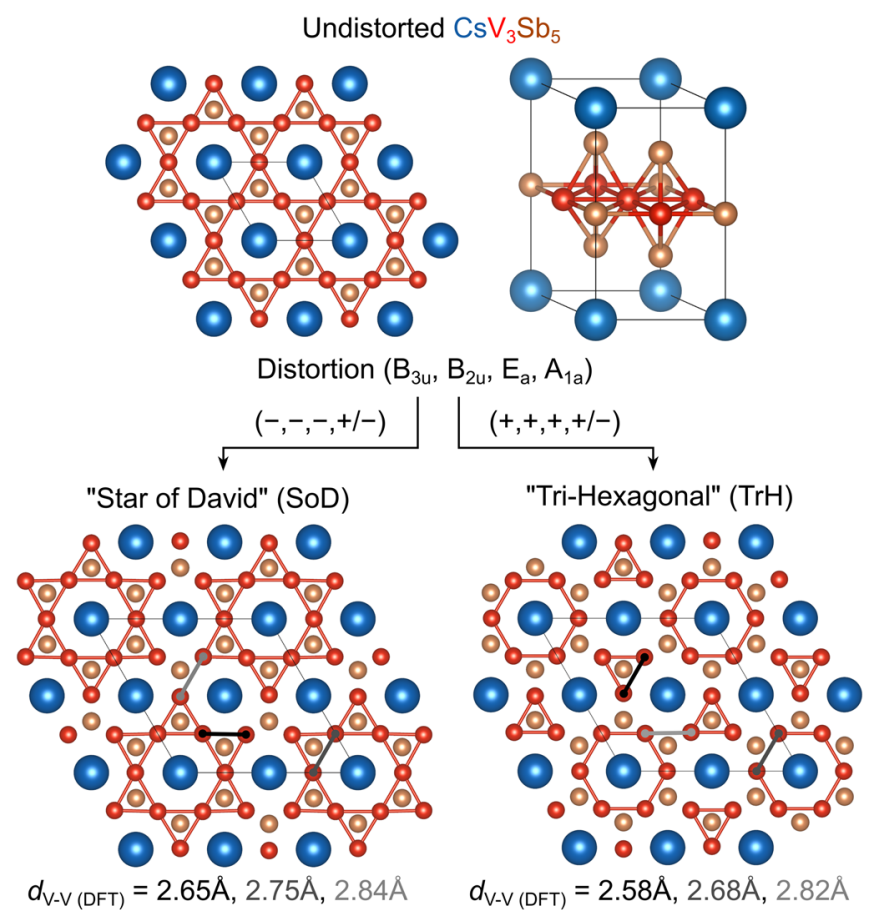

FIG. 1. $\mathrm{CsV}_{3} \mathrm{Sb}_{5}$ is a layered, exfoliatable, kagome metal consisting of a structurally perfect lattice of vanadium at room temperature. Upon cooling below $T^{*}=93 \mathrm{~K}, \mathrm{CsV}_{3} \mathrm{Sb}_{5}$ exhibits charge density wave order. A concurrent structural distortion emerges as well, which is suspected to be related to the kagome "breathing mode." Upon distortion and relaxation in both the positive and negative displacements, the breathing mode gives rise to the Star of David (SoD) and trihexagonal ( $\mathrm{TrH}$ ) candidate structures.

that the CDW instability is three dimensional in nature, with a resulting $2 \times 2 \times 4$ superstructure. Synchrotron $x$-ray data are analyzed to provide a model for the average superstructure. Together, our results provide direct evidence that the in-plane CDW is derived from vanadium orbitals which comprise the kagome lattice in $A \mathrm{~V}_{3} \mathrm{Sb}_{5}$ and validate recent efforts to map the core interactions in these materials to minimal tight-binding models built from a two-dimensional kagome network.

\section{METHODS}

\section{A. Synthesis}

Single crystals of $\mathrm{CsV}_{3} \mathrm{Sb}_{5}$ were synthesized from Cs (liquid, Alfa 99.98\%), V (powder, Sigma 99.9\%), and Sb (shot, Alfa 99.999\%). As-received vanadium powder was purified in house to remove residual oxides. Because of the extreme reactivity of elemental $\mathrm{Cs}$, all further preparation of $\mathrm{CsV}_{3} \mathrm{Sb}_{5}$ was performed in an argon glovebox with oxygen and moisture levels less than $0.5 \mathrm{ppm}$. Single crystals of $\mathrm{CsV}_{3} \mathrm{Sb}_{5}$ were synthesized using the self-flux method. The flux is a eutectic mixture of $\mathrm{CsSb}$ and $\mathrm{Cs}_{3} \mathrm{Sb}_{7}$ [37] mixed with $\mathrm{VSb}_{2}$. Elemental reagents were milled in a 
preseasoned tungsten carbide vial to form a composition that is 50 at.\% $\mathrm{Cs}_{0.4} \mathrm{Sb}_{0.6}$ eutectic.

\section{B. Electrical transport measurements}

Electronic transport measurements were performed using a Quantum Design 14 T Dynacool Physical Property Measurement System (PPMS). A Quantum Design rotator option was used to collect angle-dependent and temperaturedependent data. Crystals were exfoliated to remove any surface contaminants, and electrical contacts were made in a standard four-point geometry using gold wire and silver paint. Crystals were initially mounted such that the $c$ axis was parallel to the field (flat plates mounted flush on the resistivity stage). An alternating current of $8 \mathrm{~mA}$ and $12.2 \mathrm{~Hz}$ was driven in the $a b$ plane.

\section{Electronic structure calculations}

DFT simulations of the electronic structure of $\mathrm{CsV}_{3} \mathrm{Sb}_{5}$ unit cells were performed in VASP v5.4.4 using parameters identical to several recently reported studies $[20,21,38]$. We employed the PBE functional [39] with D3 corrections [40], a $500-\mathrm{eV}$ plane-wave energy cutoff, a $\Gamma$-centered $11 \times 11 \times 5 \mathrm{k}$ mesh, and the recommended PAW pseudopotentials for $v 5$.2. Spin-orbit coupling was activated for all calculation steps except for structural relaxation. All calculations were completed with an energy convergence cutoff of $10^{-6} \mathrm{eV}$ or better. The unit cell was relaxed, as previously described [20], with final $a$ and $c$ lattice parameters of $5.45 \AA$ and $9.35 \AA$, in good agreement with the room-temperature values determined by $x$-ray diffraction, $5.52 \AA$ and $9.36 \AA$, respectively.

WANNIER90 [41] was used to fit Wannier functions (Cs s, p; V s, p, d; Sb s, p; with a frozen fitting window $\left.E_{\mathrm{F}} \pm 2 \mathrm{eV}\right)$ and interpolate unit-cell Fermi surfaces on a $101 \times 101 \times 101$ grid. Extremal orbits were determined using SERENDIPITY [42], a new code that builds on the algorithms developed by Rourke and Julian [43] with additional symmetry and interactive visualization tools enabled by the PYTHON packages SPGLIB [44], TRIMESH [45], and PLOTLy [46].

Supercell calculations were completed on a $2 \times 2 \times 1$ supercell simulated using identical parameters and a $5 \times$ $5 \times 5 k$ mesh that was distorted along the $\mathrm{M}_{1}^{+} \mathrm{P}_{3}$ irreducible representation in ISODISTORT [47,48]. $\mathrm{M}_{1}^{+} \mathrm{P}_{3}$ included four distinct distortion modes, including $\mathrm{B}_{3 u}$ and $\mathrm{B}_{2 u} \mathrm{~V}$ sublattice modes and $\mathrm{E}_{a}$ and $\mathrm{A}_{1 a} \mathrm{Sb}_{2}$ (antimonene layer) sublattice modes. $\mathrm{Cs}$ and $\mathrm{Sb}_{1}$ (kagome layer) atomic positions were unaffected. Negative magnitudes of the $\mathrm{B}_{3 u}, \mathrm{~B}_{2 u}$, and $\mathrm{E}_{a}$ modes recreated the SoD while positive magnitudes recreated the inverse $\mathrm{TrH}$ structure. We initialized supercell structural relaxations with four different sets of mode magnitudes, $\left(\mathrm{B}_{3 u}, \mathrm{~B}_{2 u}, \mathrm{E}_{a}, \mathrm{~A}_{1 a}\right)=$ $0.3 \AA \cdot\{(+,+,+,+) ;(+,+,+,-) ;(-,-,-,+) ;(-,-,-,-)\}$, testing both the SoD and TrH structures, while additionally simulating both positive and negative magnitudes of the $\mathrm{A}_{1 a}$ mode, corresponding to $c$-axial buckling of the antimonene lattice.

Relaxation proceeded in three steps: volumetric optimization, followed by adjustment of the ionic positions, and finally, a free relaxation of the supercell lattice parameters and ionic positions simultaneously. Ultimately, the two SoD configurations relaxed to a similar structure, and the same was true for the two $\mathrm{TrH}$ structures. In the final SoD structure, we found a positive $\mathrm{A}_{1 a}$ mode, corresponding to $\mathrm{Sb}_{2}$ atoms moving further from the smaller triangles and closer to the larger triangles of the SoD structure. In the final $\mathrm{TrH}$ structure, we found that a negative $\mathrm{A}_{1 a}$ mode was favored, in which the $\mathrm{Sb}_{2}$ atoms similarly moved away from the smaller V-V triangle units. In each case, $c$-axial buckling of the $\mathrm{Sb}_{2}$ layer was minuscule, $0.005 c$ and $0.002 c$ for $\mathrm{SoD}$ and $\mathrm{TrH}$, respectively.

The SoD and $\mathrm{TrH}$ distorted supercells were energetically favored over the undistorted unit cell by $4.7 \mathrm{meV}$ and $13.5 \mathrm{meV}$ per formula unit, respectively, consistent with recently reported simulations [35]. Supercell band unfolding employed a modified version of VASPBANDUNFOLDING [49]. Unfolded Fermi surface slices in Fig. 8 were calculated on a $51 \times 51 \mathrm{BZ}$ mesh. Cubic spline interpolation was used for smoothing or upsampling prior to projecting onto the larger display range.

Fermi levels for the electronic structure calculations in Figs. 4 and 8 were determined based on prior experiments. Additional discussion is provided in the Supplemental Material [50]. Error bars obtained when extracting frequencies associated with extremal orbits of the unfolded supercells, as shown in Fig. 9, were determined by graphically selecting orbit paths clearly within a given orbit and those clearly outside and are a product of the pixel resolution of the calculations. The top of the error bar is the outer bounding area, the bottom the inner bounding area, and the average of these bounds was chosen as the nominal value. Errors in determining the extremal orbits of the parent structure are small and not shown (less than $10 \mathrm{~T}$ ).

\section{X-ray diffraction measurements}

High-dynamic-range x-ray diffraction maps were collected at the QM2 beamline at CHESS. The incident $\mathrm{x}$-ray wavelength was $\lambda=0.42755 \AA$, selected using a doublebounce diamond monochromator. Temperature was controlled by bathing the small single-crystal samples inside a stream of cold flowing helium gas. Diffraction was recorded in transmission through the sample using a 6-megapixel photon-counting pixel-array detector with a silicon sensor layer. Full 360-degree sample rotations, sliced into 0.1-degree frames, were indexed to the hightemperature crystal structure and transformed to reciprocal space. Some elements of the data reduction employed the NeXpy software package. Crystal structures were visualized in VESTA [51]. Diffraction data were analyzed within 

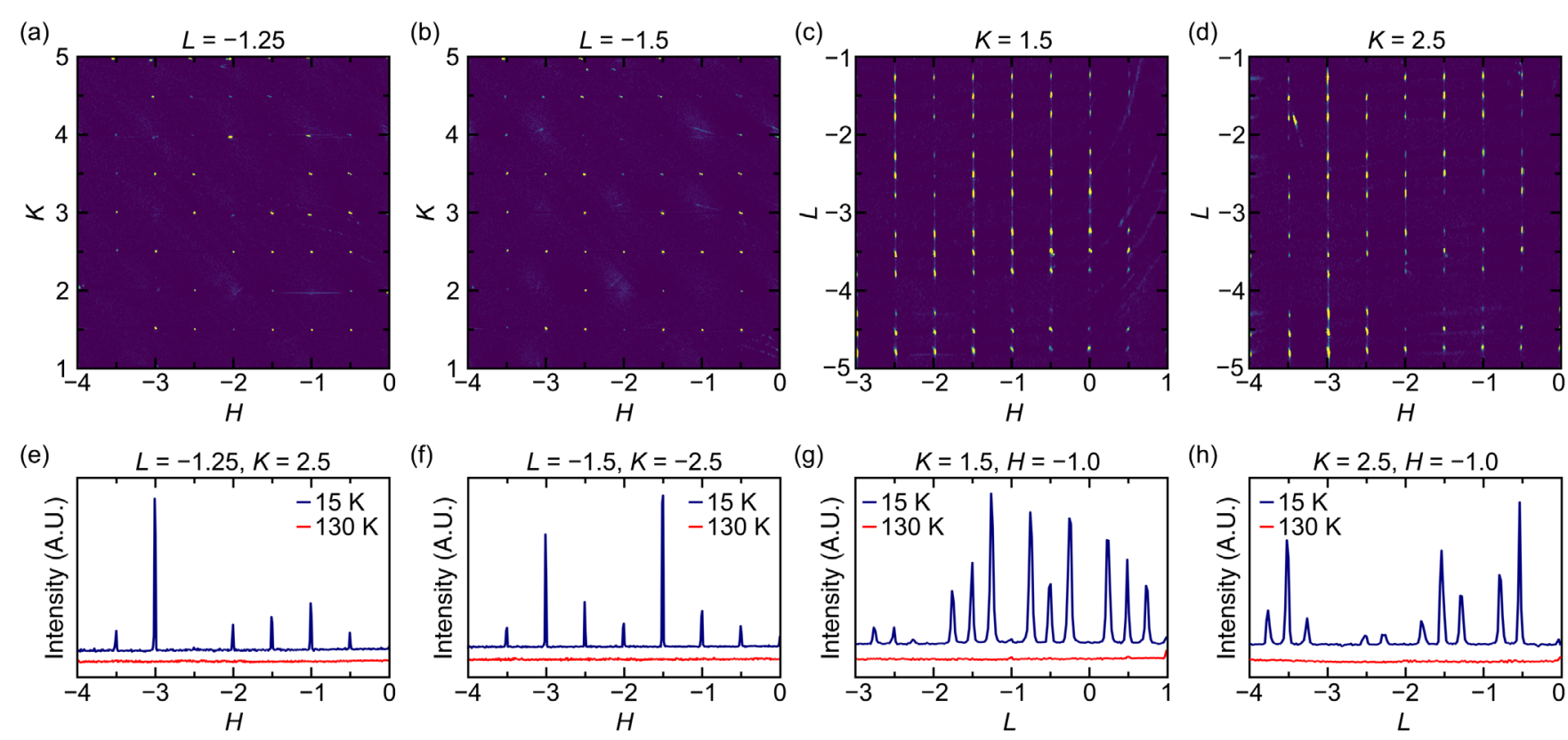

FIG. 2. (a)-(d) Two-dimensional slices through reciprocal space on half-integer Bragg planes at $15 \mathrm{~K}$, highlighting superlattice peaks in $\mathrm{CsV}_{3} \mathrm{Sb}_{5}$. (e)-(h) Line cuts through the $2 \mathrm{D}$ data, highlighting the periodicity of the superlattice peaks. Cuts performed at the base temperature of $15 \mathrm{~K}$ (blue) contrast data collected at $130 \mathrm{~K}$ (red), confirming that the superlattice peaks emerge simultaneous the charge density wave order at $T^{*} \sim 94 \mathrm{~K}$. Our data indicate that the superlattice in $\mathrm{CsV}_{3} \mathrm{Sb}_{5}$ is described by a wave vector of $(0.5,0.5,0.25)$.

the APEX3 software package, and data were corrected for absorption and extinction effects. Refinement of the structure was performed using the integrated SHELX software package [52]. Charge flipping simulations of diffraction data were performed using the TOPAZ software package [53-55].

\section{E. Second harmonic generation optical measurements}

Second harmonic generation (SHG) measurements were performed using an ultrafast laser with a pulse duration of $40 \mathrm{fs}$ and a repetition rate of $50 \mathrm{kHz}$. The laser was tuned to a center wavelength of $800 \mathrm{~nm}$ and a sample fluence of $3 \mathrm{~mJ} / \mathrm{cm}^{2}$. An oblique incidence reflection geometry was employed with both incoming and outgoing beams $P$ polarized. The reflected SHG at $400 \mathrm{~nm}$ was isolated with a spectral filter and detected using a back-illuminated CMOS image sensor. Overall, SHG intensities were extracted by averaging over the scattering plane angle. A sample-in-vacuum optical cryostat was used to cool the sample below the CDW phase transition temperature.

\section{EXPERIMENTAL RESULTS}

\section{A. Crystal structure}

The $A \mathrm{~V}_{3} \mathrm{Sb}_{5}(A: \mathrm{K}, \mathrm{Rb} \mathrm{Cs})$ family of kagome metals are layered, exfoliable materials consisting of $\mathrm{V}_{3} \mathrm{Sb}_{5}$ slabs intercalated by alkali metal cations. The vanadium sublattice forms a perfect kagome lattice under ambient conditions (Fig. 1). $\mathrm{CsV}_{3} \mathrm{Sb}_{5}$ is the terminal endpoint of the alkali-metal series; it shows the highest superconducting transition $\left(T_{c}=2.5 \mathrm{~K}\right)$ and an onset of CDW order below $T^{*}=94 \mathrm{~K}$ [21]. The $\mathrm{CDW}$ is accompanied by a weak structural distortion manifest as a superlattice of Bragg scattering in synchrotron $x$-ray diffraction data.

Early measurements within the $L=0$ scattering plane resolved only $\mathbf{q}=(0.5,0,0)$ and $(0,0.5,0)$-type superlattice reflections [20], which is seemingly at odds with recent STM reports of $2 \times 2$ supercells associated with $3 \mathbf{Q}$ charge order. To address this, an expanded exploration of superlattice peaks was conducted at finite $L$ values, with the results shown in Fig. 2. In this higher resolution data, a more complex, three-dimensional superlattice structure is observed that is best indexed by a $\mathbf{q}=(0.5,0.5,0.25)$ wave vector. Note that $(0.5,0.5)$-type superlattice reflections are largely not resolvable in the $L=0$ plane, accounting for the initial failure to index them. The in-plane component of the superlattice modulation agrees with the $3 \mathbf{Q}$ structure observed in local probes. The superlattice peaks at $(0.5,0.5$, 0.25)-type positions vanish above the CDW ordering temperature and indicate a modulation of the in-plane distortion along the $c$ axis (interplane phasing).

Considering first the in-plane distortions allowed on an idealized kagome lattice, the kagome breathing mode often leads to lower energy structures, and this mode matches preliminary conclusions drawn from STM and DFT studies of $\mathrm{KV}_{3} \mathrm{Sb}_{5}$ [18,35,36]. As shown in Fig. 1, the structure can undergo two potential breathing mode distortions: (1) the SoD distortion and (2) the $\mathrm{TrH}$ distortion. The phasing 
along the $c$ axis, which governs the modulation of the distortion motifs along the out-of-plane direction, are naively expected to be of a lower energy scale than the in-plane components. To determine the nature of the threedimensional superstructure that forms below the CDW transition, the low-temperature $(15 \mathrm{~K})$ diffraction data were refined. Approximately 30000 reflections (about 4500 unique) were indexed within a hexagonal unit cell with lattice parameters $a=b=11.05410(13) \AA, c=$ 37.334(5) $\AA$, and $\alpha=\beta=90^{\circ}, \gamma=120^{\circ}$.

SHG data indicate that inversion symmetry is not broken below the ordering transition [50], and, as a result, data were initially analyzed via charge-flipping in the $P \overline{1}$ space group. This provided a baseline visualization for distortions below the transition and, absent any further constraints, already suggests an average cell with modulation between TrH- and SoD-type distortions along the $c$ axis. Further refinement was then pursued within the space group $P \overline{3}$, assuming a minimal threefold symmetry that conformed with the diffraction data as well as inversion symmetry demonstrated from the SHG data. While the data can potentially be indexed in a lower rotational symmetry (e.g., centered orthorhombic), we could not find sufficient evidence within the current data to perform the refinement lower than $P \overline{3}$. For the solution in $P \overline{3}$, a twinning model was used, with twins realized via a twofold rotation along the $(0,0,1)$ axis. We qualitatively tested alternate hexagonal twinning models, and in all cases, the refined structure remains nearly unchanged.

Atomic positions and displacement parameters were refined in $P \overline{3} \quad(\mathrm{R} 1=0.089, \mathrm{GoF}=1.32)$, with the resulting refinement parameters provided in the Supplemental Material [50]. The resulting $P \overline{3}$ structure is shown in Fig. 3. To highlight the differences in bonding and $\mathrm{V}-\mathrm{V}$ motifs, we chose to draw $\mathrm{V}-\mathrm{V}$ bond lengths less than or equal to $2.79 \AA$. The middle panels demonstrate the different motifs, with distortions in each kagome plane highlighted. The top and bottom layers of the lattice assume an in-plane $\operatorname{TrH}$ distortion while the intervening layers assume a weak SoD-like distortion. The rightmost panels of Fig. 3 identify the distorted V-V bond distances. For graphical simplicity, bonds within $0.0025 \AA$ of their mean value were grouped and averaged. Full bonding information is available in the CIF file [50]. The TrH-like layers feature the most distinct distortion, which manifests the largest deviation in bond lengths from the parent structure. The two unique SoD-like layers are similar, though the central layer exhibits slightly weaker V-V bond distortions. We emphasize here that this is a depiction of the average structure produced by modeling the $\mathrm{x}$-ray diffraction data. More complex twinning effects or stacking disorder within the four-layer unit cell can influence the appearance of the average structure.

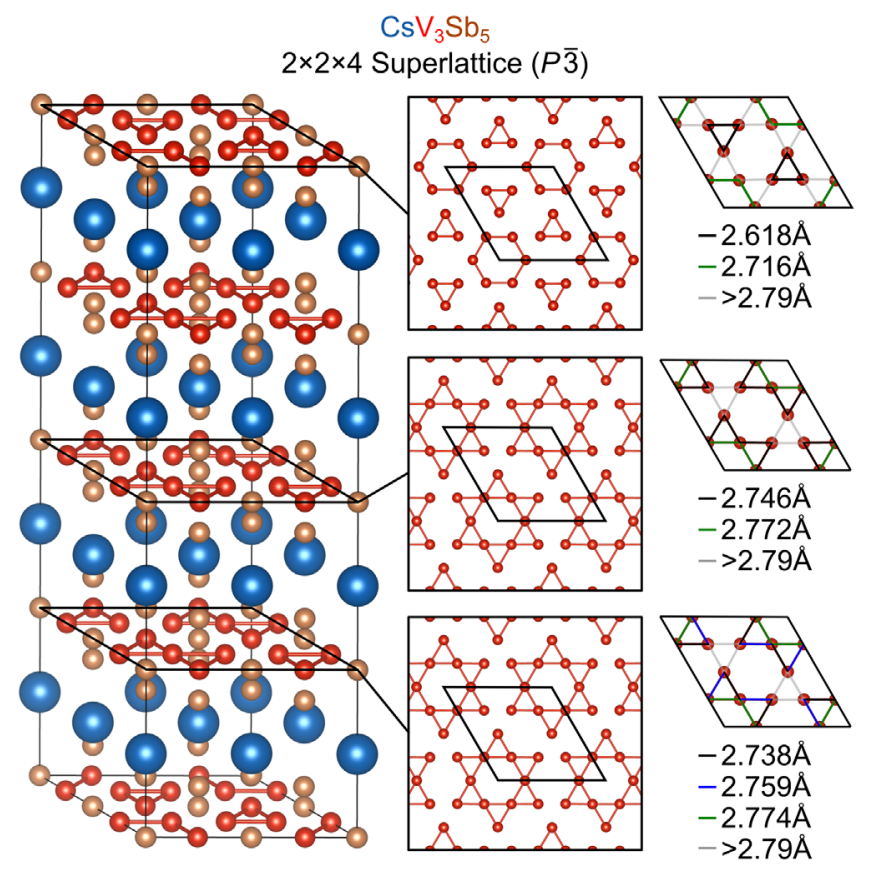

FIG. 3. Single-crystal diffraction data imply that $\mathrm{CsV}_{3} \mathrm{Sb}_{5}$ distorts into a $2 \times 2 \times 4$ supercell, where the kagome layers exhibit both TrH- and SoD-like distortions. Our current model indexes the cell in the $P \overline{3}$ space group. The SoD-like distortions are substantially weaker than the TrH-like distortion, and two unique SoD layers are noted.

\section{B. Electronic structure}

As the interlayer interactions are expected to be weak in $\mathrm{CsV}_{3} \mathrm{Sb}_{5}$, we neglect the impact of the $c$-axis component of the superlattice on the electronic structure and focus on the impact of the in-plane distortion modes. This was verified by comparing the calculated band structures of the nominal $2 \times 2 \times 1$ cell with the $2 \times 2 \times 4$ cell proposed by SCXRD [50]. Candidate structures $\left(\mathrm{M}_{1}^{+} \mathrm{P}_{3}\right.$ irrep.) matching the pure $\mathrm{TrH}$ and SoD distortions were selected for DFT relaxation and band structure calculations. Our DFT studies found that both the SoD and $\mathrm{TrH}$ distortions were slightly favored over the undistorted structure, by $4.7 \mathrm{meV} / \mathrm{f} . u$. and $13.5 \mathrm{meV} /$ f.u., respectively. DFT-relaxed structures are shown alongside the experimental, undistorted crystal structure in Fig. 1.

Despite the low stabilization energy of the distorted structures relative to the parent structure, the predicted vanadium lattice distortions are significant. The $\mathrm{V}-\mathrm{V}$ bond lengths are all an equal length $(2.72 \AA)$ in the parent structure and transform to three distinct lengths: $2.65 \AA, 2.75 \AA$, and $2.84 \AA$ for the SoD distortion, and $2.58 \AA, 2.68 \AA$, and $2.82 \AA$ for the TrH distortion. The experimentally refined structure shows slightly weaker distortions and corresponding lengths of $2.74 \AA$, $2.77 \AA$, and $2.79 \AA$ for SoD layers and $2.62 \AA, 2.72 \AA$, and $2.86 \AA$ for the TrH layers. Concurrently, the Sb graphitic sublattice is fragmented into individual hexagons and also 

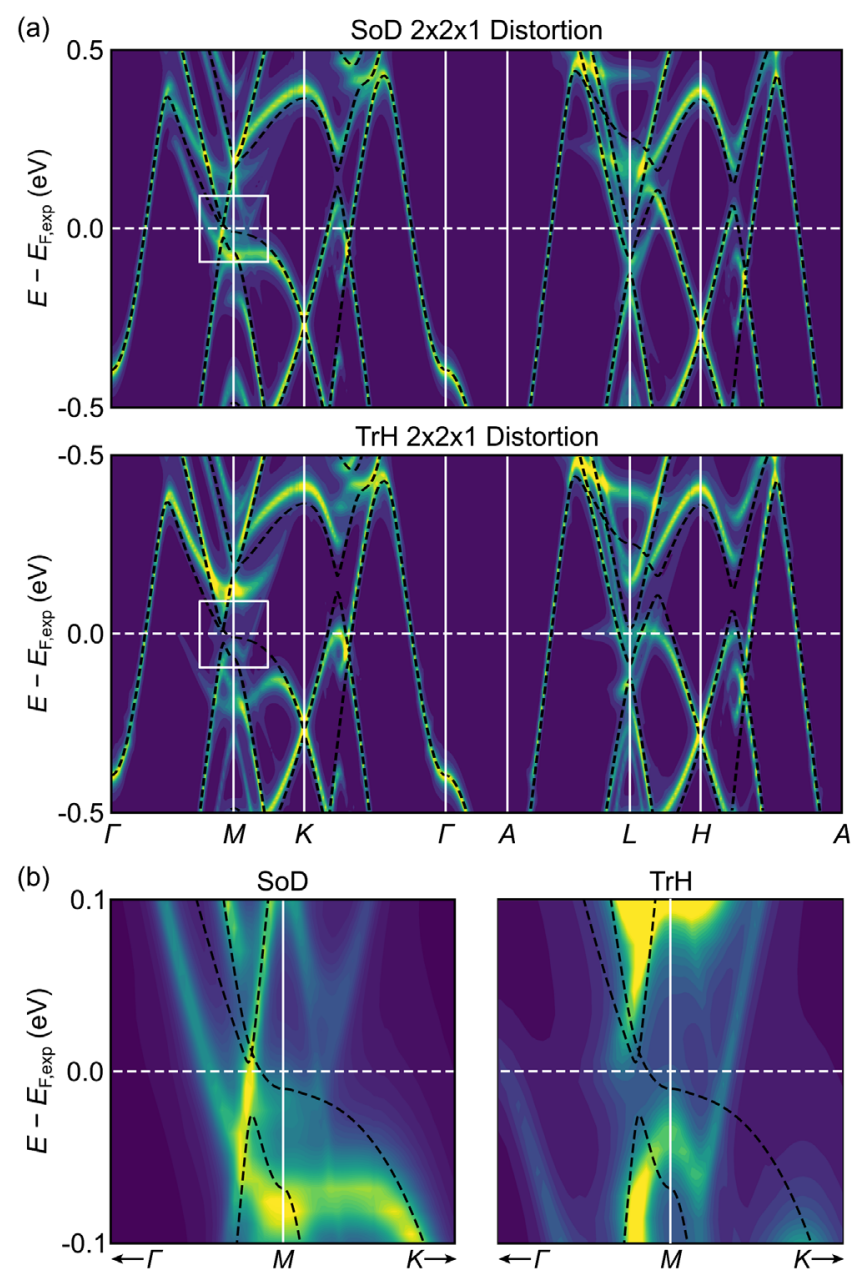

FIG. 4. (a) Unfolded electronic structure of the SoD and TrH distortions in $\mathrm{CsV}_{3} \mathrm{Sb}_{5}$ with the undistorted electronic structure superimposed for comparison (black). The largest perturbation to the structure appears near the Dirac-like bands near M. (b) Closeup of the changes near the $\mathrm{M}$ point, highlighting the new bands that appear as a result of the CDW and the associated structural distortion.

hosts a slight buckling in the $c$ direction for both distorted structure types.

Figure 4 shows the effect of the two superlattice types ( $\mathrm{SoD}$ and $\mathrm{TrH}$ ) on the $a b$ initio electronic structure of $\mathrm{CsV}_{3} \mathrm{Sb}_{5}$. The resulting band diagrams were unfolded for comparison to the undistorted electronic structure shown in previous works $[17,20]$. The heat map shows the relative projections of the electronic states after the unfolding, and the black dashed lines represent the undistorted band structure of $\mathrm{CsV}_{3} \mathrm{Sb}_{5}$. In the low-temperature distorted state, the electronic structure is largely unperturbed, particularly the central band about $\Gamma$, which derives from the $\mathrm{Sb} p$ orbitals. However, the bands near the M point, which are the relevant Dirac-like bands associated with the vanadium $d$ orbitals, are altered significantly.

Figure 4(b) shows expanded views of the SoD and $\mathrm{TrH}$ electronic structures in the vicinity of the $M$ points. An orbital decomposed band diagram ("orbital bands") further identifies these states as originating primarily from vanadium orbitals [50]. The emergence of the CDW and the resulting superlattice therefore has a clear effect on the electronic structure near $E_{\mathrm{F}}$ for the vanadium orbitals comprising the Dirac-like crossings. This effect has a significant impact on the Fermi surface and is expected to impact transport sensitive to topographical changes in the electronic structure at $E_{\mathrm{F}}$. Experimental detection of these effects is discussed in the next section.

\section{Quantum oscillation measurements}

An effective bulk probe of the low-energy band structure is the measurement of quantum oscillations in high-field electron transport measurements. Crystals of the $A \mathrm{~V}_{3} \mathrm{Sb}_{5}$ kagome metals are high mobility metals with low residual resistivity (about $0.1 \mu \Omega-\mathrm{cm}$ ) values [17,21,32], rendering quantum oscillation measurements an appealing probe for exploring the low-temperature electronic structure.

Figure 5 presents a series of temperature-dependent quantum oscillation measurements on stoichiometric crystals of $\mathrm{CsV}_{3} \mathrm{Sb}_{5}$ with a residual-resistivity-ratio (RRR) of 80 . The crystals were mounted with the $c$ axis parallel to the magnetic field while the current was driven within the $a b$ plane. The normal "background" magnetoresistance (MR) was modeled using a power law function $\rho_{0} \approx \alpha H^{\beta}+\gamma$ fit over the range from $4 \mathrm{~T}$ to $14 \mathrm{~T}$. The oscillatory component of the MR was then isolated by subtracting the background MR $\Delta \rho(H)=\rho(H)-\rho_{0}(H)$. Figure 5(a) shows the oscillatory component of the magnetoresistance as a function of temperature and field, and oscillations are seen to persist up to $25 \mathrm{~K}$. The oscillation pattern is relatively complex, with multiple harmonics visible by inspection.

Quantum oscillation data collected at $1.8 \mathrm{~K}$ are isolated in Fig. 5(b), where multiple frequency components have been noted by Greek letters. At higher fields, we further highlight several regions that show contributions from additional, higher-frequency oscillations in Fig. 5(c). Because of the presence of multiple closely spaced frequencies (discussed in the next paragraph), we have grouped similar frequency components together in this initial inspection of the subtracted data. All frequencies persist between different measurements and different crystals.

Turning first to the low-frequency spectrum, Fig. 5(d) shows the Fourier transform of the data at multiple temperatures with $H \leq 250 \mathrm{~T}$. Four well-defined frequencies appear $(\alpha, \beta, \gamma, \delta)$. At higher frequencies with $400<$ $H<2000 \mathrm{~T}$, the Fourier transform in Fig. 5(e) shows an additional five well-defined frequencies $(\epsilon, \kappa, \lambda, \mu, \eta)$. The peak designated $\epsilon$ technically appears as two sharp peaks; however, this effect is likely extrinsic, and we currently consider the $\epsilon$ peak as the average of these two peaks.

While the modes above $250 \mathrm{~T}$ vanish quickly with increasing temperature above $2 \mathrm{~K}$, the low-frequency modes remain well defined up to $25 \mathrm{~K}$. The temperature 


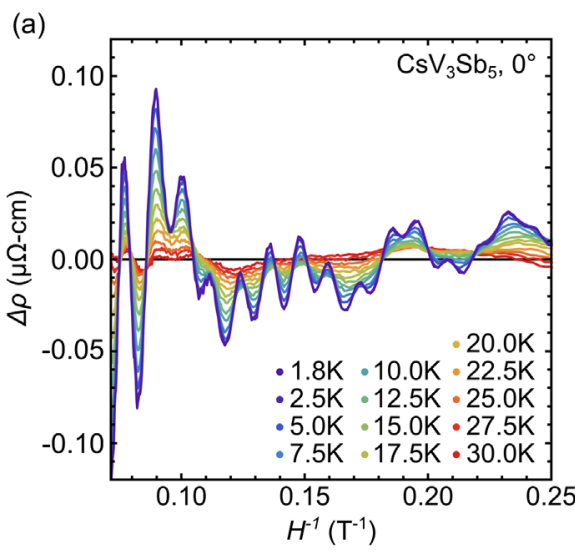

(d)

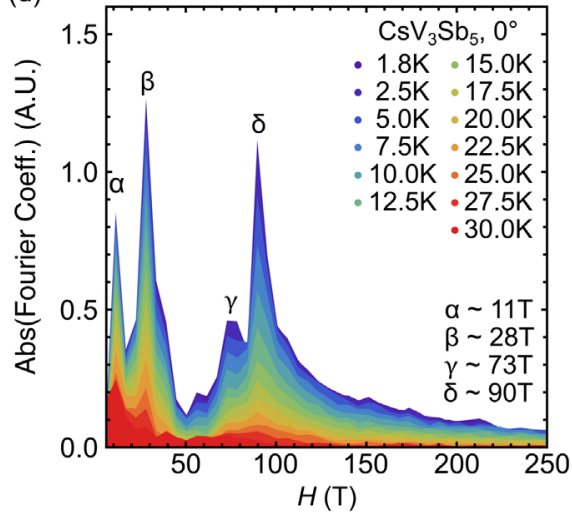

(b)

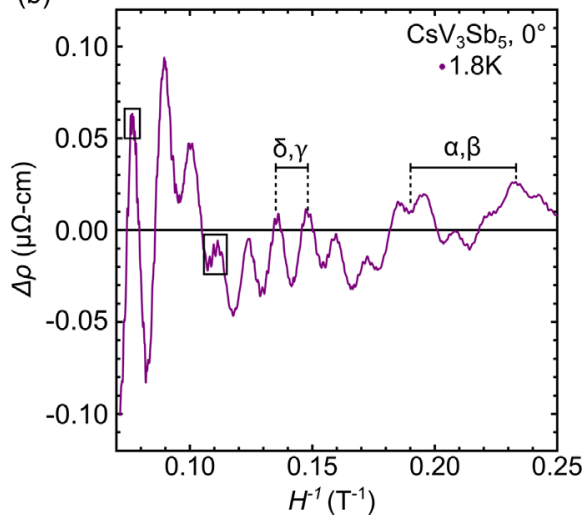

(e)

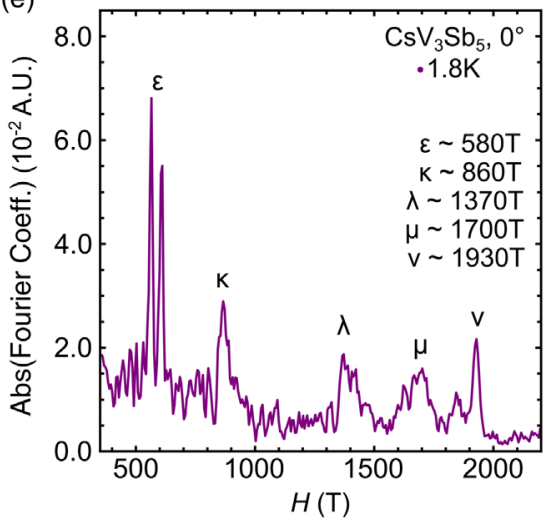

(c)

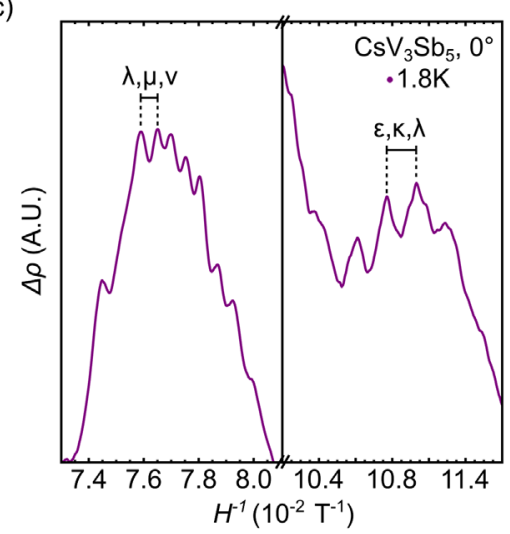

(f)

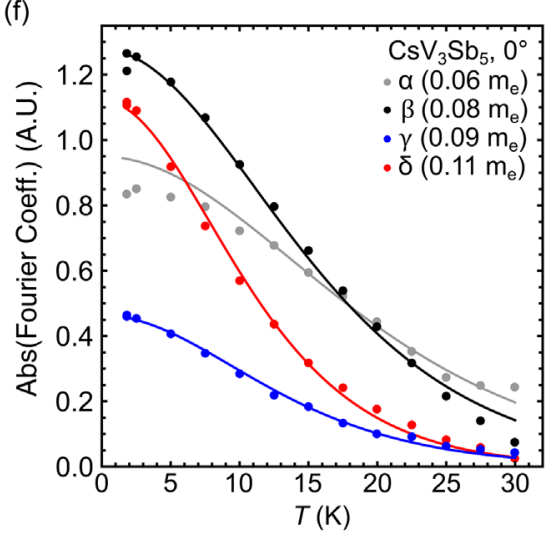

FIG. 5. (a) Oscillatory component of the magnetoresistance extracted from the temperature-dependent SdH data collected on single crystals of $\mathrm{CsV}_{3} \mathrm{Sb}_{5}$ mounted with the $\mathrm{c}$ axis parallel to the magnetic field $\left(0^{\circ}\right)$. (b) A high-resolution scan at $1.8 \mathrm{~K}$ shows significant contributions from high-frequency modes, particularly at the peaks and troughs of the general oscillatory behavior. (c) Magnified view of the high-frequency features, providing visual confirmation for multiple high-frequency modes. (d,e) Fourier transformation of the quantum oscillation data, showing the low- and high-frequency components of the power spectrum. The nine unique frequencies have been assigned greek letters. (f) Temperature dependence of the Fourier coefficients is used in conjunction with the Lifshitz-Kosevich (LK) formula to extract the cyclotron "effective masses." All low-frequency modes show very low effective masses, consistent with transport originating from the Dirac-like crossings at M.

dependence of the Fourier coefficients of the $\alpha, \beta, \gamma$, and $\delta$ orbits are shown in Fig. 5(f). The cyclotron "effective mass" $\left(m_{\mathrm{eff}}^{*}\right)$ can be extracted using the approximate Lifshitz-Kosevich (LK) form $a_{i}(T) \approx X /(B \sinh X / B)$, where $X=\alpha m_{\mathrm{eff}}^{*} T$. Here, $B$ is the magnetic flux density and is typically selected as the mean field within the FFT window. The parameter $\alpha$ is a constant defined as $14.69 \mathrm{~T} / \mathrm{K}$. The resulting $m_{\mathrm{eff}}^{*}$ values are low for these low-frequency orbits-nearly $1 / 10$ of the free electron mass - consistent with transport originating from the Dirac modes expected near the $\mathrm{M}$ point.

Whereas the temperature dependence of the quantum oscillations provides information regarding the scattering, lifetime, and effective mass of the carriers, the angular dependence can provide information regarding the topography of the Fermi surface. Figure 6(a) presents a series of angle-dependent quantum oscillation measurements collected at $1.8 \mathrm{~K}$ where $\theta=0^{\circ}$ denotes the $c$ axis parallel to the $H$ field. The Fourier transforms of the data in Fig. 6(a) are shown in Fig. 6(b), and the frequencies of the $\delta$ and $\beta$ orbits are plotted as a function of angle in Fig. 6(c). The $\alpha$ and $\gamma$ orbits shift and quickly convolve into neighboring frequencies with increasing angle, precluding their analysis at finite $\theta$.

Conceptually, the orbits that generate the oscillations can be imagined as slices through the Fermi surface at different approach angles. "Extremal" cross sections with the largest and smallest cross-sectional areas will generate distinct oscillation frequencies. For example, a perfectly spherical Fermi pocket exhibits no angular dependence and only one frequency from the circular cross section. A strongly anisotropic pocket (e.g., those from 2D Dirac cones) would show a strong dependence with angle, as oblique slices through a cylinder become progressively larger as the angle increases. The sharp upturns seen above $40^{\circ}$ in the $\delta$ and $\gamma$ orbits are consistent with orbits derived from strongly anisotropic pockets, and-as we will demonstrate in the next section-are best ascribed to electrons within Dirac-like features associated with the vanadium kagome lattice. 

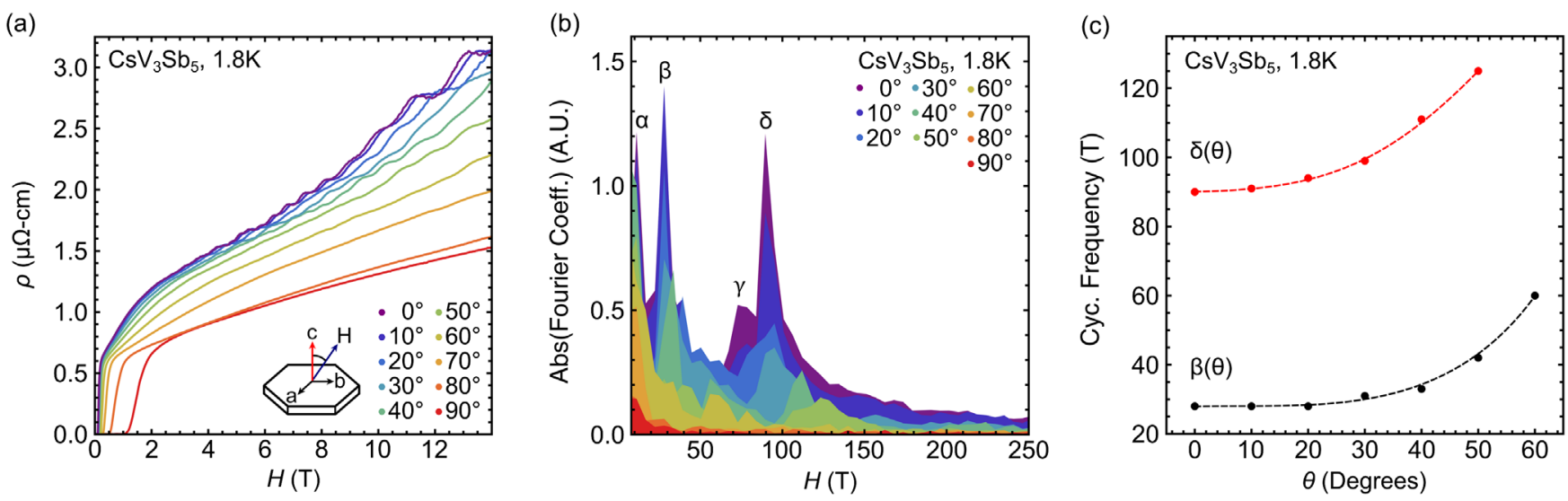

FIG. 6. (a) Raw electronic resistivity as a function of field and angle, where the angle is defined as being between the $c$ axis and the magnetic field. Oscillations are clearly visible at greater than $2 \mathrm{~T}$. The oscillations appear to be dampened with angle, vanishing for $\theta>60^{\circ}$. (b) Fourier transform of angle-resolved $\mathrm{SdH}$ data, showing primary low-frequency contributions to the power spectrum. The high-frequency contributions are suppressed rapidly by rotation. (c) Two of the most prominent frequencies $(\beta, \delta)$ are shown as a function of rotation angle. Dashed lines serve as a guide to the eye. The frequencies were estimated from (b) using Gaussian functions to approximate both the broadening and shortening of peaks.

\section{Fermi surface topography and frequency correlation}

The transport data shown in Figs. 5 and 6 reveal a complex superposition of quantum oscillations originating from multiple portions of the Fermi surface. In order to identify how the CDW and the associated crystallographic distortions perturb the Fermi surface, the oscillation frequencies (i.e., enclosed Fermi surface pockets) seen experimentally must be correlated to the DFT-calculated Fermi surfaces. To do so, we first examine the undistorted Fermi surface in the context of the possible extremal orbits.

To determine the orbits accurately, the Fermi energy needs to be well defined. It is worth taking a moment to review the spread of $E_{\mathrm{F}}$ 's reported in the current literature, as the Dirac-like nature of the bands near $E_{\mathrm{F}}$ renders rapid changes in the sizes of electron pockets with relatively minor shifts in Fermi energy. Initial DFT studies $[17,18,20,21,32]$ found Fermi levels slightly below those determined experimentally by ARPES $[10,20]$ and STM measurements $[18,19]$. For simplicity, we refer to these earlier results as $E_{F, \text { lit }}$ and $E_{F, \exp }$, respectively. Recent DFT studies have since provided self-consistent results closer to experimental values [35]; we refer to these values as $E_{F, \mathrm{DFT}}$, which agree with the self-consistent DFT calculations presented in this work.

Figure 7 shows the calculated Fermi surface using the undistorted $\mathrm{CsV}_{3} \mathrm{Sb}_{5}$ structure where $E_{\mathrm{F}}=E_{F, \text { exp }}$. Despite already demonstrating that the underlying electronic structure is perturbed by the CDW, it is nevertheless instructive to first understand the undistorted Fermi surface. The orbits identified by SERENDIPITY are shown in Fig. 7(a) in gray. We provide 2D slices of the Fermi surface at $k_{z}=0$ and $k_{z}=0.5$ for a more convenient comparison since all of the extremal orbits at $E_{F, \exp }$ occur on these two highsymmetry planes. The Fermi surface maps reveal a variety of possible extremal orbits, confirming that the quantum oscillations in $\mathrm{CsV}_{3} \mathrm{Sb}_{5}$ should contain multiple frequencies, though the $9329 \mathrm{~T}$ and $12846 \mathrm{~T}$ frequencies are significantly above the experimental range of detection in Figs. 5(d), 5(e), and 6(b).

First, considering the measurable high frequencies $(250 \mathrm{~T}<f<2000 \mathrm{~T}$ ), we find only four extremal orbits (B-I, 699 T; A-I, 1123 T; A-II, 1831 T; and C-II, 1967 T) to match five measured values $(\varepsilon \approx 580 \mathrm{~T}, \kappa \approx 860 \mathrm{~T}$, $\lambda \approx 1370 \mathrm{~T}, \mu \approx 1700 \mathrm{~T}$, and $\nu \approx 1930 \mathrm{~T}$ ). At lower frequencies, the agreement is much worse: Only one calculated low-frequency orbit $(\mathrm{C}-\mathrm{I}, 39 \mathrm{~T})$ is found to compare with four low-frequency oscillations observed in experiments $(\alpha \approx 11 \mathrm{~T}, \beta \approx 28 \mathrm{~T}, \gamma \approx 74 \mathrm{~T}$, and $\delta \approx 90 \mathrm{~T})$. A table of the calculated (DFT) frequencies at $E_{\mathrm{F}}=E_{F, \exp }$, along with the associated cyclotron masses $m_{\text {cyc }}$, has been included in the Supplemental Material (Fig. 3) [50].

Given the significant CDW band reconstruction presented in Fig. 4, a low-temperature Fermi surface modification in $\mathrm{CsV}_{3} \mathrm{Sb}_{5}$ is expected. Figure 8 displays unfolded Fermi surface slices for pure $\mathrm{SoD}$ and $\operatorname{TrH} 2 \times 2 \times 1$ supercells at $E_{F, \text { exp }}$, demonstrating this reconstruction. While the central $\mathrm{Sb} p$ orbits are largely unaffected by the CDW, $\mathrm{V} d$-bands gap and change the Fermi surface. On the $k_{z}=0.0$ plane, the single, large B-II orbit reconstructs into small orbits in both structures, generating triangular orbits around the $K$ points at the corners of the Brillouin zone. On the $k_{z}=0.5$ plane, the A-II central pocket and the smaller B-I orbit around $K$ are largely unaffected by the $\mathrm{CDW}$; however, the larger C-II orbit (dashed line) is strongly affected. In the SoD structure, this orbit is 

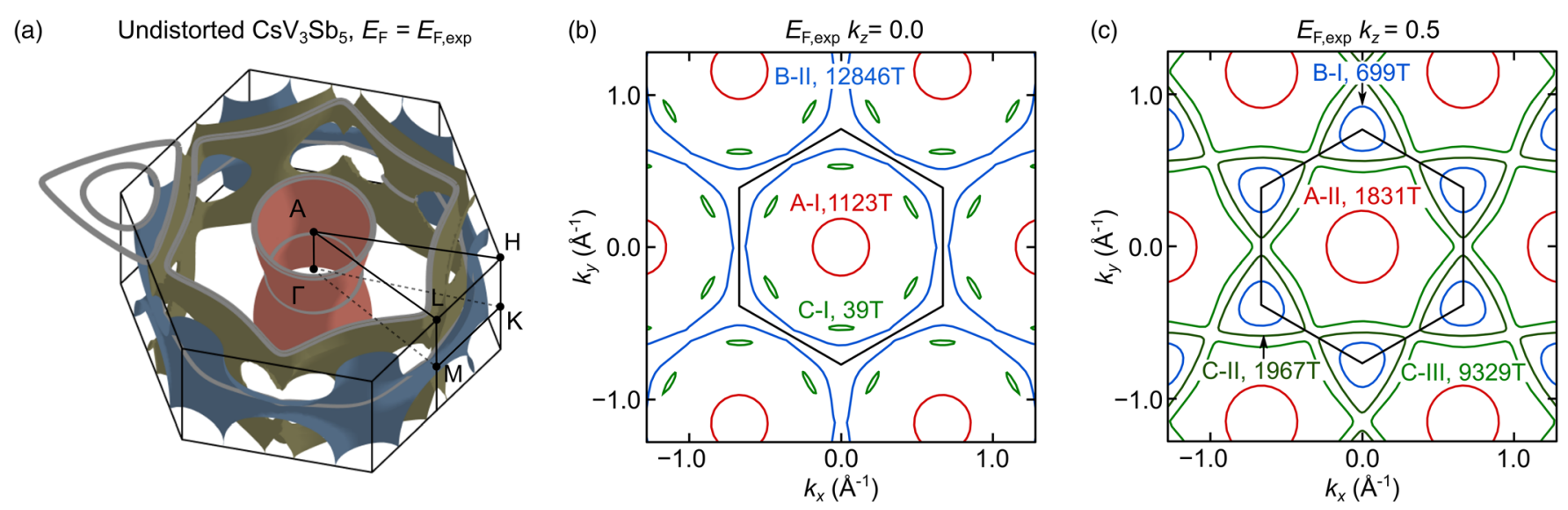

FIG. 7. (a) Fermi surface for undistorted $\mathrm{CsV}_{3} \mathrm{Sb}_{5}$ calculated with $E_{\mathrm{F}}=E_{F \text {,exp }}$, which shows a variety of potential orbits. Orbits calculated with the SERENDIPITY PYTHON package are shown highlighted in gray. For clarity, in panels (b) and (c), we show isoenergy contours at $k_{z}=0$ and $k_{z}=0.5$ for the $E_{\mathrm{F}}=E_{F, \text { exp }}$ surface with all unique extremal orbits marked. Several high- and low-frequency orbits can be identified. The Fermi surface pocket of origin is indicated through the orbit color, which will be used for comparison throughout our discussion. While there are clearly multiple frequencies in the predicted spectrum, note that there is only one symmetry unique (39 T) low-frequency mode, which is at odds with our experimental observations. Further, for frequencies $400<f<2000 \mathrm{~T}$, there are only four predicted modes, as opposed to the five experimentally observed components.

completely gapped out at $E_{F, \text { exp }}$, while in the TrH structure, the C-II orbit persists.

Supporting these models, prior ARPES results show the A-I and A-II orbits as well as the B-I orbit in $\mathrm{KV}_{3} \mathrm{Sb}_{5}$ and $\mathrm{CsV}_{3} \mathrm{Sb}_{5}$ both above and below the CDW transition $[18,20]$. STM results at lower temperatures also show the preservation of the A-I and A-II orbits and are consistent with at least one triangular orbit at the $K$ points [19]. Therefore, with regard to the preservation of the A-I, A-II, and B-I orbits, CDW calculations in both SoD and $\mathrm{TrH}$ structures appear to be consistent with experimental results to date. STM data also validate the reconstruction of the B-II orbit captured within the DFT models; however, our models also predict numerous other changes in the lowfrequency (small-orbit) regime.

Investigating the band reconstruction in the distorted state further, the data in Fig. 8(a) clearly show additional features. Figure 8(b) provides a simplified, pictorial representation of the Fermi surface and closed orbits in the undistorted and $\operatorname{TrH}$ structures to aide discussion. All possible orbits that exist in the undistorted structure in Fig. 8(a) are depicted at $E_{F, \text { exp }}$. Orbits that are too large to be experimentally observed with our current data are shown in gray. The remaining orbits are color-coded, consistent with the pocket designations shown previously in Fig. 7. For this qualitative comparison, we focus on the orbits within the TrH structure for two reasons: (1) The additional modes are less obvious in the 2D data for the $\operatorname{TrH}$ (but no less relevant), and (2) as we will show, the $\operatorname{TrH}$ structure produces one additional frequency in the "mid-frequency" regime, which makes the presence of layers with this configuration distinguishable.
Focusing on comparison of the high-frequency orbits, we see that there are a total of four experimentally accessible orbits at $E_{F \text {,exp }}$ in the undistorted structure. Upon introducing the $\mathrm{TrH}$ distortion, several key changes occur. In the $k_{z}=0$ plane, the distortion generates three distinct vanadium $d$ orbits at $E_{F, \text { exp }}$ by shifting and gapping bands around the $M$ and $K$ points near the corners and sides of the zone. Notably, band reconstruction about the $\mathrm{M}$ point forms a new medium-frequency triangular orbit at $E_{F \text {,exp }}$. The $k_{z}=0.5$ plane, in contrast, is largely preserved in the new configuration, with the exception of the addition of one Dirac-like orbit at the $L$ point.

Thus, in this high-frequency regime, the $\mathrm{TrH}$ distortion has several effects: (1) the generation of three additional Dirac-like modes, (2) the preservation of high-frequency orbits primarily comprised of Sb states, and (3) the introduction of a new triangular orbit from the band reconstruction. It is worth noting that other, smaller Dirac-like orbits are likely present, but the resolution of the present supercell calculation limits our search to orbits greater than $30 \mathrm{~T}$.

Figure 9 summarizes all the numerical data characterizing orbits in the undistorted and distorted SoD and TrH Fermi surfaces and overlays these with the experimentally observed quantum oscillations. For the undistorted structure, an exhaustive search was performed for all extremal orbits within a range of $E_{\mathrm{F}}$ spanning from above and below the Fermi levels reported throughout the literature thus far $\left(E_{F, \text { lit }}-E_{F, \exp }-E_{F, \mathrm{DFT}}\right)$. For the distorted structures, the calculated frequencies are shown at $E_{F}=E_{F, \text { exp }}$. Orbits are again colored to remain consistent with their pocket designations in previous 

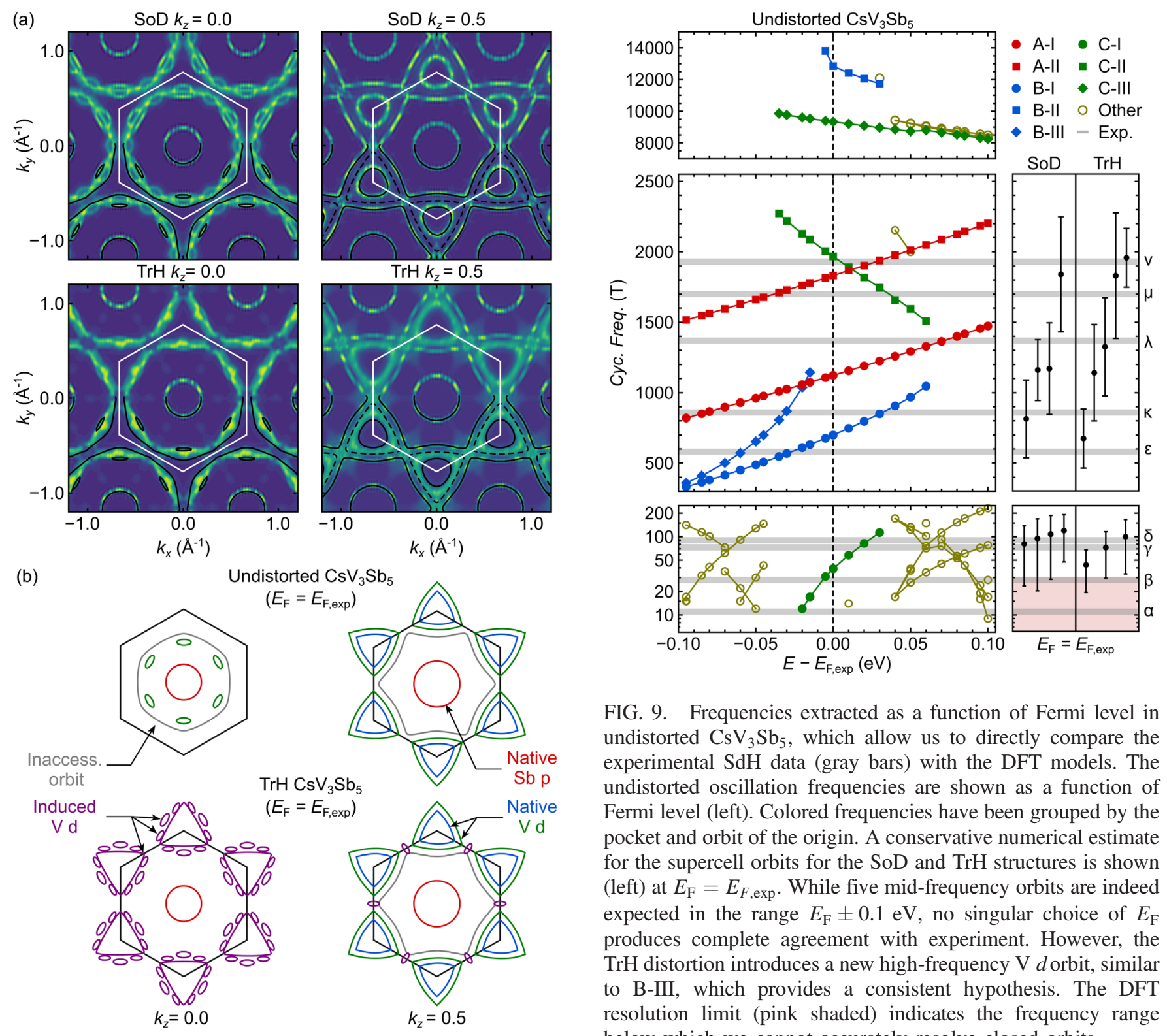

FIG. 8. (a) Fermi surfaces for the SoD and TrH distortions in $\mathrm{CsV}_{3} \mathrm{Sb}_{5}$ generated from the unfolded supercell band structures. Undistorted Fermi surface (black lines) contours are shown for comparison. A significant reconstruction of the $\mathrm{V} d$ states occurs. Most high-frequency modes are preserved, though the C-II orbit (dashed line) is gapped in the SoD structure. (b) Schematic description of Fermi surface reconstruction. Gray orbits are well above measurable frequencies. Band reconstruction in the $\mathrm{TrH}$ supercell introduces one additional triangular orbit around the $\mathrm{M}$ point while preserving higher-frequency native $\mathrm{V} d$ and $\mathrm{Sb} p$ orbits. Notably, the distortion also introduces three, smaller, Dirac-like orbits (purple) shown in Fig. 4, consistent with our measurements.

figures. For completeness, we also show orbits up to $15000 \mathrm{~T}$, though these orbits are not resolvable in the current experiments. The experimentally observed frequencies are overlaid as horizontal gray bars.

FIG. 9. Frequencies extracted as a function of Fermi level in undistorted $\mathrm{CsV}_{3} \mathrm{Sb}_{5}$, which allow us to directly compare the experimental $\mathrm{SdH}$ data (gray bars) with the DFT models. The undistorted oscillation frequencies are shown as a function of Fermi level (left). Colored frequencies have been grouped by the pocket and orbit of the origin. A conservative numerical estimate for the supercell orbits for the $\mathrm{SoD}$ and $\mathrm{TrH}$ structures is shown (left) at $E_{\mathrm{F}}=E_{F, \text { exp }}$. While five mid-frequency orbits are indeed expected in the range $E_{\mathrm{F}} \pm 0.1 \mathrm{eV}$, no singular choice of $E_{\mathrm{F}}$ produces complete agreement with experiment. However, the $\mathrm{TrH}$ distortion introduces a new high-frequency $\mathrm{V} d$ orbit, similar to B-III, which provides a consistent hypothesis. The DFT resolution limit (pink shaded) indicates the frequency range below which we cannot accurately resolve closed orbits.

First examining comparisons of the models in the highfrequency regime $(200<f<3000 \mathrm{~T})$, there are a total of four possible (symmetry unique) orbits at $E_{F, \exp }$. These are the same four shown in the schematic representation of the undistorted structure. There is one orbit (B-III) that appears at slightly lower $E_{\mathrm{F}}$, though it is gapped by $E_{F \text {,exp. }}$. Comparison of the experimental frequencies and all three models (at $E_{\mathrm{F}}=E_{F, \text { exp }}$ ) shows that, while precise quantitative agreement between all the frequencies varies between orbits, $\operatorname{TrH}$ layers are the only qualitative match to five frequencies in this regime. In contrast, because of the loss of the C-II orbit, the SoD distortion only recovers four of the five modes. More detailed comparisons for $E_{\mathrm{F}}$ shifted away from $E_{F, \text { exp }}$ will be discussed in Sec. IV.

Next, examining the low-frequency $(f<200 \mathrm{~T})$ regime, the experimental data identifies at least four well-defined 
frequencies about $E_{F, \exp }$. However, at $E_{F, \exp }$, there is only one orbit in the undistorted structure. This orbit (C-I) is predominantly comprised of vanadium $d$ orbitals associated with the Dirac-like crossings along the $\Gamma-K$ line. However, as shown throughout this work, substantial reconstruction is expected about the $\mathrm{M}$ point. Characterization of the well-defined, closed orbits in the supercell models is shown to the right of the low-frequency (undistorted) structure panel. There are four orbits in the SoD model and three in the $\operatorname{TrH}$ model. However, it is important to note that our unfolded supercell calculations are unable to resolve orbits with frequencies less than $30 \mathrm{~T}$.

\section{DISCUSSION}

While precise numerical agreement between quantum oscillation frequencies and DFT-derived oscillation frequencies at $E_{F, \text { exp }}$ is lacking in all single-layer structures modeled, the presence of the $\operatorname{TrH}$ structure at $E_{F, \exp }$ within the unit cell is the only means of capturing the multiplicity of orbits within the experimentally accessible frequency windows. This is consistent with our x-ray diffraction data, which identifies $\mathrm{TrH}$ distorted planes within the structure. However, the modulation between $\mathrm{TrH}$ and SoD distorted planes along the $c$ axis likely renders reality as a more complex convolution of the orbits calculated within single-layer models. Further work computationally modeling the much larger $2 \times 2 \times 4$ supercell with full spin-orbit coupling is required to definitely assess the impact of this modulated supercell and generate a more quantitative comparison to the experimentally observed orbital frequencies.

Irrespective of the out-of-plane modulation, when combined with DFT models, our quantum oscillation data demonstrate that the reconstructed electronic states near the Fermi level are intimately tied to the vanadium $d$ orbitals. This is particularly true for the M points, which are relevant for their contributions to the topologically protected surface states. Specifically, the observation of multiple low-frequency orbits provides direct evidence of a CDW-derived reconstruction of vanadium bands endemic to the underlying kagome lattice in $\mathrm{CsV}_{3} \mathrm{Sb}_{5}$. This finding agrees with recent ARPES results, which identify gapping around the states at the M points [56].

When matching ARPES or other Fermi-surface sensitive probes, it is worthwhile to consider the impact of choosing alternative Fermi levels in the single-layer DFT models (i.e., away from $E_{F, \text { exp }}$ ), in the comparison between models and the data. In the low-frequency regime for the undistorted structure, multiple orbits appear when moving both above and below $E_{F, \text { exp }}$, mimicking the multiple modes found in the experiment; however, this scenario can be precluded with the following arguments: (1) For the case where $E_{\mathrm{F}}$ lies below $E_{F, \exp }$-in the regime where multiple low-frequency orbits appear-the multiplicity of the highfrequency modes does not match the data, as the C-II mode is absent. (2) For the case where $E_{\mathrm{F}}$ lies above $E_{F \text {,exp }}$, the
C-II and B-I orbits are quickly gapped out in the distorted structures, leaving no explanation for the five midfrequency oscillations.

Superlattice reflections with a propagation wave vector $\mathbf{q}=(0.5,0.5,0.25)$ in $\mathrm{x}$-ray scattering data indicating a $2 \times 2 \times 4$ superstructure with a correlation length matching the native crystallinity of the sample. Primary Bragg reflections in the undistorted state are anisotropic because of $c$-axis broadening, and the superlattice reflections show the same degree of anisotropy. This indicates a minimum correlation length of approximately $200 \AA$ for the out-of-plane superlattice modulation, which is born from the poorer interplane crystallinity. While the in-plane wave vector $(h, k)=(0.5,0.5)$ matches the $3 \mathbf{Q}$ structure observed in STM, the out-of-plane component of $\mathbf{q}$ implies a four-unit-cell phasing along the $c$ axis. The average structure refined in $P \overline{3}$ suggests a modulation of distortion types along the $c$ axis. While there are a number of possible stacking sequences of SoD and $\mathrm{TrH}$ structures, the solution presented here almost falls naturally out of charge flipping in $P \overline{1}$ and is further sharpened by refining the structure within $P \overline{3}$. Future work resolving the presence of orthorhombic twins in the bulk is necessary to justify pursuing lower-symmetry structures or more complex combinations of motifs (e.g., phased offsets between layers).

The $2 \times 2 \times 4$ unit cell resolved in $\mathrm{CsV}_{3} \mathrm{Sb}_{5}$ seemingly contrasts the $2 \times 2 \times 2$ cell identified in $\mathrm{KV}_{3} \mathrm{Sb}_{5}$ [18]. Future diffraction studies will be required to fully explore this apparent difference; however, one potential reason is the poorer $c$-axis crystallinity of the $\mathrm{KV}_{3} \mathrm{Sb}_{5}$ crystal explored in our earlier study. This broadening along $L$ can potentially mask $q_{L}=0.25$-type reflections, or the enhanced disorder can modify the structural ground state. Recently, a work appeared by Li et al. [57] which reported a $2 \times 2 \times 2$ superstructure in $\mathrm{CsV}_{3} \mathrm{Sb}_{5}$. Larger surveys of reciprocal space in our data reveal the 1/4-type $c$-axis superlattice reflections. While disorder within a crystal can disrupt the longer wavelength stacking and preclude its observation, random stacking faults are unable to create the smaller $q$ periodicity observed in our data. Note that $q_{L}=0.25$-type peaks have been observed in multiple crystals and at multiple $x$-ray energies in our measurements. Finally, work by Chen et al. [33] focusing on the anomalous Hall effect in $\mathrm{CsV}_{3} \mathrm{Sb}_{5}$ also appeared recently. They report the Fourier spectrum of the low-frequency orbits in quantum oscillation data but do not report the high-frequency orbits reported in our data. This could be a consequence of crystal quality, or smoothing or aliasing considerations during data collection.

\section{CONCLUSION}

Combined DFT modeling, high-resolution x-ray scattering, and quantum oscillation measurements demonstrate that the $\mathrm{CDW}$ state in $\mathrm{CsV}_{3} \mathrm{Sb}_{5}$ derives from the reconstruction of the kagome-plane vanadium orbitals with an accompanying out-of-plane modulation of the distorted 
structure. The in-plane component of the resulting $2 \times 2 \times 4$ superstructure is best modeled using the kagome breathing mode, with the $\mathrm{SoD}$ and $\mathrm{TrH}$ patterns emerging as energetically favorable structures. X-ray diffraction data are best fit via a model of modulated SoD and $\operatorname{TrH}$ distortions along the $c$ axis of the average structure. Quantum oscillation measurements provide a bulk probe of the electronic structure that demonstrates the CDW's reconstruction of the Fermi surface. They show the dominant role of vanadium orbitals within the kagome planes in $\mathrm{CsV}_{3} \mathrm{Sb}_{5}$ in the CDW and support theoretical approaches drawn from minimal models focused on the kagome substructure in $\mathrm{AV}_{3} \mathrm{Sb}_{5}$ superconductors.

\section{ACKNOWLEDGMENTS}

S.W. gratefully acknowledges discussions with Leon Balents, Binghai Yan, and Ziqiang Wang. We gratefully thank Matthew Benning and Michael Ruf of Bruker Corporation for their assistance in the integration of synchrotron data with Apex3 and their crystallography discussions. This work was supported by the National Science Foundation (NSF) through Enabling Quantum Leap: Convergent Accelerated Discovery Foundries for Quantum Materials Science, Engineering and Information (Q-AMASE-i): Quantum Foundry at UC Santa Barbara (DMR-1906325). This research made use of the shared facilities of the NSF Materials Research Science and Engineering Center at UC Santa Barbara (DMR1720256). The UC Santa Barbara MRSEC is a member of the Materials Research Facilities Network. B. R. O. and P.M.S. also acknowledge support from the California NanoSystems Institute through the Elings Fellowship program. S. M. L.T. was supported by the National Science Foundation Graduate Research Fellowship Program under Grant No. DGE-1650114. This work is based upon research conducted at the Center for High Energy X-ray Sciences (CHEXS), which is supported by the National Science Foundation under Grant No. DMR-1829070. We acknowledge use of the shared computing facilities of the Center for Scientific Computing at UC Santa Barbara, supported by NSF CNS-1725797.

[1] D. E. Freedman, T. H. Han, A. Prodi, P. Müller, Q.-Z. Huang, Y.-S. Chen, S. M. Webb, Y. S. Lee, T. M. McQueen, and D. G. Nocera, Site Specific X-Ray Anomalous Dispersion of the Geometrically Frustrated Kagome Magnet, Herbertsmithite, $\mathrm{ZnCu}_{3}(\mathrm{OH})_{6} \mathrm{Cl}_{2}$, J. Am. Chem. Soc. 132, 16185 (2010).

[2] D. Wulferding, P. Lemmens, P. Scheib, J. Röder, P. Mendels, S. Chu, T. Han, and Y.S. Lee, Interplay of Thermal and Quantum Spin Fluctuations in the Kagome Lattice Compound Herbertsmithite, Phys. Rev. B 82, 144412 (2010).
[3] T. Han, S. Chu, and Y. S. Lee, Refining the Spin Hamiltonian in the Spin-1/2 Kagome Lattice Antiferromagnet Using Single Crystals, Phys. Rev. Lett. 108, 157202 (2012).

[4] M. Fu, T. Imai, T.-H. Han, and Y.S. Lee, Evidence for a Gapped Spin-Liquid Ground State in a Kagome Heisenberg Antiferromagnet, Science 350, 655 (2015).

[5] T.-H. Han, J. S. Helton, S. Chu, D. G. Nocera, J. A. Rodriguez-Rivera, C. Broholm, and Y.S. Lee, Fractionalized Excitations in the Spin-Liquid State of a Kagome-Lattice Antiferromagnet, Nature (London) 492, 406 (2012).

[6] W.-S. Wang, Z.-Z. Li, Y.-Y. Xiang, and Q.-H. Wang, Competing Electronic Orders on Kagome Lattices at van Hove Filling, Phys. Rev. B 87, 115135 (2013).

[7] S. V. Isakov, S. Wessel, R. G. Melko, K. Sengupta, and Y. B. Kim, Hard-Core Bosons on the Kagome Lattice: ValenceBond Solids and Their Quantum Melting, Phys. Rev. Lett. 97, 147202 (2006).

[8] A. O'Brien, F. Pollmann, and P. Fulde, Strongly Correlated Fermions on a Kagome Lattice, Phys. Rev. B 81, 235115 (2010).

[9] A. Rüegg and G. A. Fiete, Fractionally Charged Topological Point Defects on the Kagome Lattice, Phys. Rev. B 83, 165118 (2011).

[10] S. Yan, D. A. Huse, and S. R. White, Spin Liquid Ground State of the $S=1 / 2$ Kagome Heisenberg Antiferromagnet, Science 332, 1173 (2011).

[11] H.-M. Guo and M. Franz, Topological Insulator on the Kagome Lattice, Phys. Rev. B 80, 113102 (2009).

[12] W.-H. Ko, P. A. Lee, and X.-G. Wen, Doped Kagome System as Exotic Superconductor, Phys. Rev. B 79, 214502 (2009).

[13] S.-L. Yu and J.-X. Li, Chiral Superconducting Phase and Chiral Spin-Density-Wave Phase in a Hubbard Model on the Kagome Lattice, Phys. Rev. B 85, 144402 (2012).

[14] M. L. Kiesel, C. Platt, and R. Thomale, Unconventional Fermi Surface Instabilities in the Kagome Hubbard Model, Phys. Rev. Lett. 110, 126405 (2013).

[15] K. Barros, J. W. Venderbos, G.-W. Chern, and C. Batista, Exotic Magnetic Orderings in the Kagome Kondo-Lattice Model, Phys. Rev. B 90, 245119 (2014).

[16] X. Feng, K. Jiang, Z. Wang, and J. Hu, Chiral Flux Phase in the Kagome Superconductor $\mathrm{AV}_{3} \mathrm{Sb}_{5}$, Sci. Bull. 66, 1384 (2021).

[17] B. R. Ortiz, L. C. Gomes, J. R. Morey, M. Winiarski, M. Bordelon, J. S. Mangum, I. W. Oswald, J. A. RodriguezRivera, J. R. Neilson, S. D. Wilson et al., New Kagome Prototype Materials: Discovery of $\mathrm{KV}_{3} \mathrm{Sb}_{5}, \mathrm{RbV}_{3} \mathrm{Sb}_{5}$, and $\mathrm{CsV}_{3} \mathrm{Sb}_{5}$, Phys. Rev. Mater. 3, 094407 (2019).

[18] Y.-X. Jiang, J.-X. Yin, M. M. Denner, N. Shumiya, B. R. Ortiz, J. He, X. Liu, S. S. Zhang, G. Chang, I. Belopolski et al., Discovery of Topological Charge Order in Kagome Superconductor $\mathrm{KV}_{3} \mathrm{Sb}_{5}$, Nat. Mater. 20, 1353 (2021).

[19] H. Zhao, H. Li, B. R. Ortiz, S. M. Teicher, T. Park, M. Ye, Z. Wang, L. Balents, S. D. Wilson, and I. Zeljkovic, Cascade of Correlated Electron States in a Kagome Superconductor $\mathrm{CsV}_{3} \mathrm{Sb}_{5}$, arXiv:2103.03118.

[20] B. R. Ortiz, S. M. Teicher, Y. Hu, J. L. Zuo, P. M. Sarte, E. C. Schueller, A. M. Abeykoon, M. J. Krogstad, S. Rosenkranz, R. Osborn, R. Seshadri, L. Balents, J. He, 
and S. D. Wilson, $\mathrm{CsV}_{3} \mathrm{Sb}_{5}: A \mathbb{Z}_{2}$ Topological Kagome Metal with a Superconducting Ground State, Phys. Rev. Lett. 125, 247002 (2020).

[21] B. R. Ortiz, E. Kenney, P. M. Sarte, S. M. Teicher, R. Seshadri, M. J. Graf, and S. D. Wilson, Superconductivity in the $\mathbb{Z}_{2}$ Kagome Metal $\mathrm{KV}_{3} \mathrm{Sb}_{5}$, Phys. Rev. Mater. 5, 034801 (2021).

[22] Z. Liang, X. Hou, W. Ma, F. Zhang, P. Wu, Z. Zhang, F. Yu, J.-J. Ying, K. Jiang, L. Shan et al., Three-Dimensional Charge Density Wave and Robust Zero-Bias Conductance Peak inside the Superconducting Vortex Core of a Kagome Superconductor $\mathrm{CsV}_{3} \mathrm{Sb}_{5}$, Phys. Rev. X 11, 031026 (2021).

[23] H. Chen, H. Yang, B. Hu, Z. Zhao, J. Yuan, Y. Xing, G. Qian, Z. Huang, G. Li, Y. Ye et al., Roton Pair Density Wave and Unconventional Strong-Coupling Superconductivity in a Topological Kagome Metal, arXiv:2103.09188.

[24] Q. Yin, Z. Tu, C. Gong, Y. Fu, S. Yan, and H. Lei, Superconductivity and Normal-State Properties of Kagome Metal $\mathrm{RbV}_{3} \mathrm{Sb}_{5}$ Single Crystals, Chin. Phys. Lett. 38, 037403 (2021).

[25] W. Duan, Z. Nie, S. Luo, F. Yu, B. R. Ortiz, L. Yin, H. Su, F. Du, A. Wang, Y. Chen, X. Lu, J. Ying, S. D. Wilson, X. Chen, Y. Song, and H. Yuan, Nodeless Superconductivity in the Kagome Metal $\mathrm{CsV}_{3} \mathrm{Sb}_{5}$, Sci. China-Phys. Mech. Astron. 64, 107462 (2021).

[26] H.-S. Xu, Y.-J. Yan, R. Yin, W. Xia, S. Fang, Z. Chen, Y. Li, W. Yang, Y. Guo, and D.-L. Feng, Multiband Superconductivity with Sign-Preserving Order Parameter in Kagome Superconductor $\mathrm{CsV}_{3} \mathrm{Sb}_{5}$, Phys. Rev. Lett. 127, 187004 (2021).

[27] C. Mu, Q. Yin, Z. Tu, C. Gong, H. Lei, Z. Li, and J. Luo, S-Wave Superconductivity in Kagome Metal $\mathrm{CsV}_{3} \mathrm{Sb}_{5}$ Revealed by ${ }^{121 / 123} \mathrm{Sb}$ NQR and ${ }^{51} \mathrm{~V}$ NMR Measurements, Chin. Phys. Lett. 38, 077402 (2021).

[28] C. C. Zhao, L. S. Wang, W. Xia, Q. W. Yin, J. M. Ni, Y. Y. Huang, C. P. Tu, Z. C. Tao, Z. J. Tu, C. S. Gong, H. C. Lei, Y. F. Guo, X. F. Yang, and S. Y. Li, Nodal Superconductivity and Superconducting Domes in the Topological Kagome Metal $\mathrm{CsV}_{3} \mathrm{Sb}_{5}$, arXiv:2102.08356.

[29] Y. Gu, Y. Zhang, X. Feng, K. Jiang, and J. Hu, Gapless Excitations inside the Fully Gapped Kagome Superconductors $\mathrm{AV}_{3} \mathrm{Sb}_{5}$, arXiv:2108.04703.

[30] F. Du, S. Luo, B. R. Ortiz, Y. Chen, W. Duan, D. Zhang, X. Lu, S. D. Wilson, Y. Song, and H. Yuan, Pressure-Tuned Interplay between Charge Order and Superconductivity in the Kagome Metal $\mathrm{KV}_{3} \mathrm{Sb}_{5}$, Phys. Rev. B 103, L220504 (2021).

[31] K. Chen, N. Wang, Q. Yin, Z. Tu, C. Gong, J. Sun, H. Lei, Y. Uwatoko, and J.-G. Cheng, Double Superconducting Dome and Triple Enhancement of $\mathrm{T}_{c}$ in the Kagome Superconductor $\mathrm{CsV}_{3} \mathrm{Sb}_{5}$ under High Pressure, Phys. Rev. Lett. 126, 247001 (2021).

[32] S.-Y. Yang, Y. Wang, B. R. Ortiz, D. Liu, J. Gayles, E. Derunova, R. Gonzalez-Hernandez, L. Smejkal, Y. Chen, S.S. Parkin et al., Giant, Unconventional Anomalous Hall Effect in the Metallic Frustrated Magnet Candidate, $\mathrm{KV}_{3} \mathrm{Sb}_{5}$, Sci. Adv. 6, eabb6003 (2020).

[33] F. Yu, T. Wu, Z. Wang, B. Lei, W. Zhuo, J. Ying, and X. Chen, Concurrence of Anomalous Hall Effect and Charge
Density Wave in a Superconducting Topological Kagome Metal, Phys. Rev. B 104, 041103 (2021).

[34] E. M. Kenney, B. R. Ortiz, C. Wang, S. D. Wilson, and M. J. Graf, Absence of Local Moments in the Kagome Metal $\mathrm{KV}_{3} \mathrm{Sb}_{5}$ as Determined My Muon Spin Spectroscopy, J. Phys. Condens. Matter 33, 235801 (2021).

[35] H. Tan, Y. Liu, Z. Wang, and B. Yan, Charge Density Waves and Electronic Properties of Superconducting Kagome Metals, Phys. Rev. Lett. 127, 046401 (2021).

[36] E. Uykur, B. Ortiz, S. Wilson, M. Dressel, and A. Tsirlin, Optical Detection of Charge-Density-Wave Instability in the Non-magnetic Kagome Metal $\mathrm{KV}_{3} \mathrm{Sb}_{5}$, arXiv:2103.07912.

[37] J. Sangster and A. Pelton, The Cs-Sb (Cesium-Antimony) System, J. Phase Equilib. 18, 382 (1997).

[38] Y. Wang, S. Yang, P. K. Sivakumar, B. R. Ortiz, S. M. Teicher, H. Wu, A. K. Sirvastava, C. Garg, D. Liu, S. S. Parkin, E. Toberer, T. McQueen, S. D. Wilson, and M. N. Ali, Proximity-Induced Spin-Triplet Superconductivity and Edge Supercurrent in the Topological Kagome Metal, $\mathrm{K}_{1-x} \mathrm{~V}_{3} \mathrm{Sb}_{5}$, arXiv:2012.05898.

[39] J. P. Perdew, K. Burke, and M. Ernzerhof, Generalized Gradient Approximation Made Simple, Phys. Rev. Lett. 77, 3865 (1996).

[40] S. Grimme, S. Ehrlich, and L. Goerigk, Effect of the Damping Function in Dispersion Corrected Density Functional Theory, J. Comput. Chem. 32, 1456 (2011).

[41] A. A. Mostofi, J. R. Yates, G. Pizzi, Y.-S. Lee, I. Souza, D. Vanderbilt, and N. Marzari, An Updated Version of wannier90: A Tool for Obtaining Maximally-Localised Wannier Functions, Comput. Phys. Commun. 185, 2309 (2014).

[42] SERENDIPITY is in development, pending an open-source release. Interested parties can contact Samuel Teicher: steicher@ucsb.edu.

[43] P. Rourke and S. Julian, Numerical Extraction of de Haasvan Alphen Frequencies from Calculated Band Energies, Comput. Phys. Commun. 183, 324 (2012).

[44] A. Togo and I. Tanaka, Spglib: A Software Library for Crystal Symmetry Search, arXiv:1808.01590.

[45] M. Dawson-Haggerty, trimesh, retrieved from https://github .com/mikedh/trimesh.

[46] Plotly Technologies, Inc., Plotly, retrieved from https://plot .ly.

[47] H. T. Stokes, D. M. Hatch, and B. J. Campbell, ISODISTORT, ISOTROPY Software Suite, retrieved from https://iso .byu.edu.

[48] B. J. Campbell, H. T. Stokes, D. E. Tanner, and D. M. Hatch, ISODISPLACE: A Web-Based Tool for Exploring Structural Distortions, J. Appl. Crystallogr. 39, 607 (2006).

[49] Q. Zheng, Vasp band unfolding, retrieved from https:// github.com/QijingZheng/VaspBandUnfolding.

[50] See Supplemental Material at http://link.aps.org/ supplemental/10.1103/PhysRevX.11.041030 for further details.

[51] K. Momma and F. Izumi, VESTA3 for Three-Dimensional Visualization of Crystal, Volumetric and Morphology Data, J. Appl. Crystallogr. 44, 1272 (2011).

[52] G. M. Sheldrick, Crystal Structure Refinement with SHELXL, Acta Crystallogr. C 71, 3 (2015). 
[53] G. Oszlányi and A. Sütő, Ab Initio Structure Solution by Charge Flipping, Acta Crystallogr. Sect. A 60, 134 (2004).

[54] G. Oszlányi and A. Sütô, Ab Initio Structure Solution by Charge Flipping. II. Use of Weak Reflections, Acta Crystallogr. Sect. A 61, 147 (2005).

[55] A. Coelho, A Charge-Flipping Algorithm Incorporating the Tangent Formula for Solving Difficult Structures, Acta Crystallogr. Sect. A 63, 400 (2007).

[56] Z. Wang, S. Ma, Y. Zhang, H. Yang, Z. Zhao, Y. Ou, Y. Zhu, S. Ni, Z. Lu, H. Chen, K. Jiang, L. Yu, Y. Zhang, X. Dong,
J. Hu, H.-J. Gao, and Z. Zhao, Distinctive Momentum Dependent Charge-Density-Wave Gap Observed in $\mathrm{CsV}_{3} \mathrm{Sb}_{5}$ Superconductor with Topological Kagome Lattice, arXiv:2104.05556

[57] H. Li, T. Zhang, Y.-Y. Pai, C. Marvinney, A. Said, T. Yilmaz, Q. Yin, C. Gong, Z. Tu, E. Vescovo et al., Observation of Unconventional Charge Density Wave without Acoustic Phonon Anomaly in Kagome Superconductors $\mathrm{AV}_{3} \mathrm{Sb}_{5}(\mathrm{~A}=\mathrm{Rb}, \mathrm{Cs})$, Phys. Rev. X 11, 031050 (2021). 